\title{
Treatment and Outcomes of Urethral Recurrence of Urinary Bladder Cancer in Women after Radical Cystectomy and Orthotopic Neobladder: A Series of 12 Cases
}

\author{
Jan Hrbáček ${ }^{\mathrm{a}}$ Petr Macek ${ }^{\mathrm{b}}$ Bedeir Ali-El-Dein ${ }^{\mathrm{c}}$ George N. Thalmann ${ }^{\mathrm{d}}$ \\ Arnulf Stenzl $^{\text {e }}$ Marek Babjuk $^{a}$ Atallah A. Shaaban ${ }^{c}$ Georgios Gakis ${ }^{\mathrm{e}}$ \\ a Department of Urology, 2nd Faculty of Medicine, Charles University and Motol University Hospital, and \\ ${ }^{b}$ Department of Urology, 1st Faculty of Medicine, Charles University and General University Hospital, Prague, \\ Czech Republic; ' Urology and Nephrology Center, Mansoura University, Mansoura, Egypt; ${ }^{\mathrm{d}}$ Department of Urology, \\ University of Bern, Bern, Switzerland; ${ }^{e}$ Department of Urology, Eberhard-Karls University, Tübingen, Germany
}

\author{
Key Words \\ Bladder cancer · Cystectomy · Female · Relapse · \\ Treatment outcome · Urethra
}

\begin{abstract}
Introduction: The incidence, treatment, and outcome of urethral recurrence (UR) after radical cystectomy (RC) for muscle-invasive bladder cancer with orthotopic neobladder in women have rarely been addressed in the literature. $\mathbf{P a}$ tients and Methods: A total of 12 patients (median age at recurrence: 60 years) who experienced UR after RC with an orthotopic neobladder were selected for this study from a cohort of 456 women from participating institutions. The primary clinical and pathological characteristics at RC, including the manifestation of the UR and its treatment and outcome, were reviewed. Results: The primary bladder tumors in the 12 patients were urothelial carcinoma in 8 patients, squamous cell carcinoma and adenocarcinoma in 1 patient each, and mixed histology in 2 patients. Three patients (25\%) had lymph node-positive disease at RC. The median time from RC to the detection of UR was 8 months (range 4-55).
\end{abstract}

Eight recurrences manifested with clinical symptoms and 4 were detected during follow-up or during a diagnostic workup for clinical symptoms caused by distant metastases. Treatment modalities were surgery, chemotherapy, radiotherapy, and bacillus Calmette-Guérin urethral instillations. Nine patients died of cancer. The median survival after the diagnosis of UR was 6 months. Conclusions: UR after RC with an orthotopic neobladder in females is rare. Solitary, noninvasive recurrences have a favorable prognosis when detected early. Invasive recurrences are often associated with local and distant metastases and have a poor prognosis.

(c) 2014 S. Karger AG, Basel

\section{Introduction}

In recent decades, radical cystectomy $(\mathrm{RC})$ with extended pelvic lymph node dissection has become the mainstay of treatment for muscle-invasive bladder cancer. The treatment aims to provide long-term survival with an acceptable quality of life. Reconstruction of the lower urinary tract using orthotopic bladder substitution has increased the ac-

\section{KARGER 125}

C 2014 S. Karger AG, Base

0042-1138/14/0941-0045\$39.50/0 
ceptance of radical surgical treatment by both physicians and patients [1], including older age groups $[2,3]$.

The initial reluctance to perform orthotopic bladder substitution in females was due to the short urethral length (hence, the proximity of the bladder neck and bladder tumor) and the fear of incontinence after removal of the proximal urethra adjacent to the bladder neck [4]. The added morbidity of urethrectomy, the longer surgical time, and the very high rate of negative pathology made physicians question the need for routine urethrectomy in females [5]. This paved the way for a wider use of orthotopic neobladders in women. According to recent anatomical dissection studies, the external female rhabdosphincter is located in the distal half of the urethra and is innervated by the pudendal nerve from below. Smooth muscle innervated by the autonomic nerve system via branches from the pelvic plexus running alongside the lateral vaginal walls is present throughout the length of the urethra. Hence, a careful technique of bladder neck and vaginal dissection will preserve the nerve supply and muscle structures necessary for continence [6].

Retrospective pathological studies have reported a $12 \%$ (range 6-46) rate of concomitant tumor (of any stage) in the bladder and the urethra in females. Bladder neck involvement is a strong risk factor for concomitant urethral malignancy, observed in 19-33\% of urethral tumors. In contrast, urethral tumors have never been found in women with a tumor-free bladder neck. Some investigators found an association with tumor involvement of the anterior vaginal wall, but later studies failed to confirm this finding [4].

We describe the incidence, treatment, and outcomes in 12 cases of recurrence of urinary bladder cancer (UBC) in the female urethra after RC.

\section{Patients and Methods}

The 12 patients reported herein were selected from a multiinstitutional collaborative cohort of 456 women who underwent $\mathrm{RC}$ for UBC in 1 of the 5 participating centers between 1994 and 2011. The median follow-up was 64 months (range 3-196). We retrospectively reviewed the clinical and pathological records of all 456 female patients for age, histology, tumor stage and grade, lymph node involvement, soft-tissue surgical margins, final urethral margin status, and survival [7].

The intensity of the follow-up depended on each institution's protocol. Recurrence in the surgical bed was defined as local, recurrence at distant organs was defined as distant, and recurrence in the urethra was defined as urethral. Clinical outcomes were measured from the date of cystectomy to the date of the first documented recurrence, the date of death, or the date of the last follow-up if the patient had not experienced disease recurrence. A positive urethral margin was a significant risk factor for urethral recurrence (UR) [7].
The present report describes the 12 recurrent patients identified within the defined cohort, including 1 who relapsed after the end of the original study (table 1).

\section{Results}

A total of 12 patients (median age at RC: 58 years; range 41-71) were studied. The bladder tumors were urothelial carcinoma in 8 patients (67\%), squamous cell and adenocarcinoma in 1 patient $(8 \%)$ each, and mixed urothelial carcinoma in 2 patients (17\%). Three patients had lymph node-positive disease ( 2 with $\mathrm{pN} 1$ and 1 with $\mathrm{pN} 2$ ) at $\mathrm{RC}$, and none had evidence of distant metastasis at RC (cM0). Urinary diversion was performed according to Studer or Hautmann ( 6 cases each). Two patients ( $\mathrm{G}$ and $\mathrm{L}$ ) received adjuvant cisplatin-based chemotherapy after RC.

The median time from $\mathrm{RC}$ to the detection of recurrence in the urethra was 8 months (range 4-55). Eight recurrences $(67 \%)$ manifested with clinical symptoms which were pain-related in 4 patients, bleeding in 3 , gross hematuria in 2 , and urinary retention in 2 . One recurrence was seen protruding from the urethra. The remainder were detected during follow-up or during a diagnostic work-up for clinical symptoms caused either by synchronous distant metastases or by pelvic recurrence.

Treatment with curative intent was given to 6 patients, and treatment with a palliative intent to was given to 3 . Three women were unfit for or refused treatment. Open surgery was performed in 5 patients (including urinary undiversion in 3), radiotherapy was performed in 2 patients, chemotherapy was performed in 3 patients, and bacillus Calmette-Guérin (BCG) urethral instillations were used in 2 patients. Nine women (75\%) died of cancer and 3 were still alive at the time of reporting. The median survival after detection of UR was 6 months (range 2-15). The most frequent sites of distant metastases were lymph nodes and bones.

\section{Discussion}

In our study, we gathered 12 cases of UR after RC with a neobladder substitution in women. This number is too low for statistically meaningful conclusions, but several observations can be made.

The advantage of maintaining a natural voiding pattern after RC must be weighed against the risk of a subsequent tumor recurrence in the remnant urethra. An 8.1\% incidence of UR after cystoprostatectomy was reported in 
Table 1. Clinical and pathological characteristics of 12 female patients who experienced UR after RC and orthotopic neobladder

\begin{tabular}{|c|c|c|c|c|c|c|c|c|c|c|}
\hline $\begin{array}{l}\mathrm{Pa}- \\
\text { tient }\end{array}$ & RC date & $\begin{array}{l}\text { Age at } \\
\text { RC, } \\
\text { years }\end{array}$ & $\begin{array}{l}\text { Pathological } \\
\text { stage and grade } \\
\text { at RC }\end{array}$ & Histology at RC & $\begin{array}{l}\text { Neo- } \\
\text { bladder } \\
\text { type }\end{array}$ & $\begin{array}{l}\text { Time to } \\
\text { recur- } \\
\text { rence, } \\
\text { months }\end{array}$ & $\begin{array}{l}\text { Recurrence } \\
\text { detected }\end{array}$ & $\begin{array}{l}\text { Recurrence } \\
\text { stage }\end{array}$ & $\begin{array}{l}\mathrm{P} \text { or } \mathrm{M} \text { at } \\
\text { diagnosis/ } \\
\text { later in the } \\
\text { follow-up }\end{array}$ & $\begin{array}{l}\text { Time to } \\
\text { death, } \\
\text { months }\end{array}$ \\
\hline A & $29 / 05 / 1996$ & 66 & pT3a pN0 G3 & urothelial & $\mathrm{H}$ & 8 & $\mathrm{C}$ & cT3 & M & 3 \\
\hline B & $26 / 02 / 1998$ & 55 & pT2b pN0 G3 & urothelial & $\mathrm{H}$ & 4 & $\mathrm{C}$ & $\begin{array}{l}\text { cT3 cN3 } \\
\text { inoperable }\end{array}$ & M & 3 \\
\hline $\mathrm{C}$ & $27 / 02 / 2006$ & 56 & pTa pN0 G1 & urothelial & S & NR & $\mathrm{C}$ & pTX G3 & $-1-$ & - \\
\hline $\mathrm{F}$ & $30 / 03 / 2009$ & 64 & pTis + pT3b pN0 G3 & urothelial + squamous & S & 7 & $\mathrm{~F}$ & CIS & $-1-$ & - \\
\hline G & $12 / 10 / 2005$ & 66 & pTis + pT2b pN0 G2 & urothelial & $\mathrm{S}$ & 55 & $\mathrm{~W}$ & NR & $\mathrm{M}$ & 7 \\
\hline $\mathrm{H}$ & $22 / 04 / 2005$ & 63 & pT2a pN0 G2-3 & urothelial + adenocarcinoma & S & 52 & W & NR & M & 15 \\
\hline I & 23/04/1997 & 51 & pT3a pN0 G3 & adenocarcinoma & $\mathrm{H}$ & 12 & $\mathrm{C}$ & pT1 G3 & $\mathrm{P}$ & 7 \\
\hline J & $22 / 04 / 1995$ & 47 & pT3a pN1 G2 & squamous & $\mathrm{H}$ & 8 & $\mathrm{C}$ & pT1 G3 & $\mathrm{P} / \mathrm{M}$ & 7 \\
\hline $\mathrm{K}$ & $25 / 02 / 2002$ & 51 & pTis + pT3b pN0 G3 & urothelial & $\mathrm{H}$ & 13 & $\mathrm{~F}$ & pT1 G3 & $\mathrm{P}$ & NR \\
\hline
\end{tabular}

Patient A presented with bleeding, pain and acute urinary retention. At that time, she had already been receiving paclitaxel and radiotherapy for pubic bone metastases diagnosed previously. After the detection of UR, only optimal supportive care was administered. Patient B had recurrence in the urethra, which caused pain and was palpable on physical examination. During an attempted surgical removal, advanced disease with metastatic deposits was found. The urinary diversion was transformed into a Mainz pouch II. Soon thereafter, she developed a colocutaneous fistula and nephrostomy tubes had to be inserted. Patient $\mathrm{C}$ was diagnosed with UR originating in the distal urethra as detected by both ultrasonography and physical examination. After surgical removal of the recurrence, she was free of disease for the entire follow-up. Patient D had recurrence manifesting as a painful mass protruding from the urethra and causing urinary retention. Surgical treatment included removal of the vagina and neobladder infiltrated by the tumor and conversion of the urinary diversion into an ileal conduit. The recurrence histology was pT4 G3 urothelial carcinoma with myxoid features. Patient E had UR diagnosed by urethral wash-out cytology and confirmed by a biopsy. She was treated with 6 urethral BCG instillations repeated every 3-6 months. She was the only one of this series to have a positive urethral margin at the final histopathology, yet she counts among survivors, being free of disease for 45 months after the operation. Patient $F$ had her recurrence diagnosed by urethral cytology. The disease was eradicated via BCG instillations, but re- lapsed again as CIS 3 years later. Conservative management with BCG was on-going at the time of reporting. Patient $\mathrm{G}$ received adjuvant gemcitabine + cisplatin after RC. She presented with a subileus caused by UR originating from the proximal urethra and bladder neck, infiltrating the neobladder, vagina, and rectum. Surgical treatment was attempted with partial vaginal, ileal, and total rectal resection. Multiple pelvic and peritoneal metastases were discovered. The patient received palliative chemotherapy. Patient $H$ had UR detected during a work-up for an epileptic seizure caused by a synchronous brain metastasis. Radiotherapy failed to control the disease and she died with brain and pulmonary metastases. Patient I was unfit for the treatment of her urethral recurrence. Patient J presented with bleeding and gross hematuria as symptoms of squamous cell carcinoma UR. Urethrectomy was performed with conversion of the neobladder to a catheterizable continent reservoir. Nevertheless, she died of disease progression with lymph node and osseous metastases. Patient $\mathrm{K}$ was diagnosed with UR on follow-up imaging along with a concomitant pelvic mass. She was unfit for curative treatment. Patient $\mathrm{L}$ received adjuvant cisplatin-based chemotherapy after RC. UR manifested with pain and was evident on physical examination. Palliative gemcitabine and radiotherapy administration followed. $\mathrm{H}=$ Hautmann neobladder; $\mathrm{S}=$ Studer neobladder; $\mathrm{C}=$ by clinical symptoms; $\mathrm{F}=$ during follow-up; $\mathrm{W}=$ during a work-up for unrelated clinical symptoms; $\mathrm{P}=$ pelvic recurrence; $\mathrm{M}=$ distant metastasis; $\mathrm{NR}=$ not reported. a meta-analysis of unselected male patients ( 256 of 3,165 cases). UR after RC in females with an orthotopic neobladder is much less common [8]. Rates as low as $0 \%$ have been reported, albeit in small and/or short follow-up studies (table 2).

Risk factors including multifocality, primary tumor stage and grade, the presence of carcinoma in situ (CIS), and upper urinary tract involvement have been reported [5]. The most consistent association has been observed between the type of diversion and UR risk: patients with a neobladder are less likely to develop a secondary urethral tumor than those with a cutaneous diversion [9-12].

One of the reasons for the lower UR incidence in women compared to men may be the fact that squamous cell mucosa (normal or metaplastic) covers a large portion of the female urethra and the demarcation line between squamous and transitional epithelium moves cranially with increasing age [8]. Only 5 cases of UR in the retained urethra after RC with a neobladder in women have been described to date [13-17].

Generally, a positive urethral surgical margin at RC is considered to be a contraindication for neobladder and should prompt a urethrectomy [18]. Cases have been reported in the literature, however, where urethral margin positivity at the final histopathology was not associated with disease recurrence [19] or resulted in a pT0 status at re-exploration [13]. All of our patients who died of the disease had a concomitant pelvic recurrence or distant 
Table 2. UR rates in females after RC and orthotopic neobladder reported in the literature

\begin{tabular}{lrlll}
\hline Reference & $\mathrm{n}$ & $\begin{array}{l}\text { Age (range), } \\
\text { years }\end{array}$ & $\begin{array}{l}\text { Follow-up (range), } \\
\text { months }\end{array}$ & $\begin{array}{l}\text { Urethral recurrences } \\
\text { detected }\end{array}$ \\
\hline Taylor et al., 2010 [23] & 10 & $61(31-80)$ & $54(1-187)$ & 0 \\
Stein et al., 2009 [19] & 120 & $66(33-83)$ & $103(1-192)$ & $1(0.8 \%)$ \\
Akkad et al., 2006 [14] & 46 & $64(34-78)$ & $45(2-138)$ & $2(4.3 \%)$ \\
Ali-El-Dein et al., 2004 [15] & 145 & $50(23-73)$ & $17(4-78)$ & $2(1.4 \%)$ \\
Hassan et al., 2004 [11] & 29 & $63(35-88)$ & $34(1-97)$ & 0 \\
Yamashita et al., 2003 [24] & 15 & NR & $61(2-254)$ & 0 \\
Yossepowitch et al., 2003 [25] & 8 & $58(31-76)$ & $44(1-153)$ & 0 \\
Stenzl et al., 2001 [26] & 96 & $59(28-79)$ & $26(2-100)$ & 0 \\
\hline
\end{tabular}

NR $=$ Not reported

metastases at the time of UR diagnosis. Conversely, $3 \mathrm{fe}-$ males who were alive at the time of reporting had neither pelvic recurrence nor distant metastasis during followup. The primary bladder tumor stage was a predictor of survival. Of the 3 survivors among our patients, 2 had non-muscle-invasive bladder cancer at the time of RC (pTis and $\mathrm{pTa}$ ). Nine out of 10 muscle-invasive primary UBC were followed by UR with a concomitant pelvic mass and/or distant metastasis. Ultimately, all died of cancer.

Surgical removal of the recurrence with a curative intent was attempted in 5 of our 12 patients, but the disease was truly eradicated in only 1 patient (patient C). The neobladder had to be changed to a Mainz pouch II, an ileal conduit, and a catheterizable continent reservoir in patients B, D, and J, respectively. Recurrences in the form of CIS have been successfully treated with BCG instillations of the urethra; this approach was described previously by Varol et al. [20]. When chemotherapy was administered, it was done with a palliative intent. The longest survival (15 months) was observed in patient $\mathrm{H}$, who was treated with radiotherapy; the time to recurrence and the final grade 2-3 at RC suggest that her bladder cancer in general had a less aggressive character. Therefore, it seems that survival after UR treatment depends rather on the nature of the recurrence than on the treatment modality.

The oncological efficiency of follow-up in patients after RC is controversial $[21,22]$. In our 12 patients, UR were detected based on symptoms in 1 of 3 patients who were still alive at the time of reporting and in 6 of 9 patients who died of the disease. Those who died of aggressive tumors might have - or might not have - been saved by earlier detection. However, we may assume that the
2 women with urethral CIS detected at follow-up would likely have progressed over time to muscle-invasive disease had they not been diagnosed and treated.

Given the easy anatomical accessibility of the female urethra, it can easily be checked during follow-up after RC. At a minimum, the patient can be asked about a history of gross hematuria and/or pain on micturition, and a digital vaginal examination can be performed [18]. A vaginal ultrasound gives excellent resolution of the urethral region. Introital ultrasonography might be a reasonable alternative but, to the best of our knowledge, no study has addressed this issue in a systematic manner. With regard to the CIS cases in our cohort and their long-term survival, urethral wash-out cytology [20] can be considered at least for patients at risk (i.e. those with CIS in the primary UBC at RC). Because of the broad time frame (6-55 months) in which the UR developed in our patients, it is difficult to draw conclusions regarding the optimal duration and frequency of follow-up in women with orthotopic neobladders.

\section{Conclusions}

Recurrence of a bladder tumor in the female urethra after RC with an orthotopic neobladder is a rare event. Noninvasive recurrences (pTa-1 and CIS) are usually not accompanied by pelvic or distant metastasis and their prognosis is relatively favorable with adequate treatment. In contrast, if UR is associated with a local or distant disease relapse, the prognosis is dismal.
48

Urol Int 2015;94:45-49 DOI: $10.1159 / 000363112$
Hrbáček et al. 


\section{References}

$>1$ Stein JP, Lieskovsky G, Cote R, et al: Radical cystectomy in the treatment of invasive bladder cancer: long-term results in 1,054 patients. J Clin Oncol 2001;19:666-675.

-2 Roghmann F, Sukumar S, Ravi P, et al: Radical cystectomy in the elderly: national trends and disparities in perioperative outcomes and quality of care. Urol Int 2014;92:27-34.

-3 Berger I, Martini T, Wehrberger C, et al: Perioperative complications and 90-day mortality of radical cystectomy in the elderly $(75+)$ : a retrospective, multicentre study. Urol Int DOI: $10.1159 / 000357127$.

4 Stein JP, Penson DF, Wu SD, et al: Pathological guidelines for orthotopic urinary diversion in women with bladder cancer: a review of the literature. J Urol 2007;178:756-760.

$>5$ Van Poppel H, Sorgeloose T: Radical cystectomy with or without urethrectomy? Crit Rev Oncol Hematol 2003;47:141-145.

-6 Stenzl A, Höltl L: Orthotopic bladder reconstruction in women - what we have learned over the last decade. Crit Rev Oncol Hematol 2003; 47:147-154.

7 Gakis G, Ali-El-Dein B, Babjuk M, et al: A multi-institutional study on risk factors of urethral recurrence in female patients with ileal neobladder. Eur Urol Suppl 2012;11:e608a.

$>8$ Stenzl A, Bartsch G, Rogatsch H: The remnant urothelium after reconstructive bladder surgery. Eur Urol 2002;41:124-131.

-9 Stein JP, Clark P, Miranda G, et al: Urethral tumor recurrence following cystectomy and urinary diversion: clinical and pathological characteristics in 768 male patients. J Urol 2005;173:1163-1168.
10 Boorjian SA, Kim SP, Weight CJ, et al: Risk factors and outcomes of urethral recurrence following radical cystectomy. Eur Urol 2011; 60:1266-1272.

11 Hassan JM, Cookson MS, Smith JA Jr, et al: Urethral recurrence in patients following orthotopic urinary diversion. J Urol 2004;172: 1338-1341.

12 Nieder AM, Sved PD, Gomez P, et al: Urethral recurrence after cystoprostatectomy: implications for urinary diversion and monitoring. Urology 2004;64:950-954.

13 Sherwood JB, Sagalowsky AI: The diagnosis and treatment of urethral recurrence after radical cystectomy. Urol Oncol 2006;24:356361.

14 Akkad T, Gozzi C, Deibl M, et al: Tumor recurrence in the remnant urothelium of females undergoing radical cystectomy for transitional cell carcinoma of the bladder: long-term results from a single center. J Urol 2006;175:1268-1271.

15 Ali-el-Dein B, Abdel-Latif M, Ashamallah A et al: Local urethral recurrence after radical cystectomy and orthotopic bladder substitution in women: a prospective study. J Urol 2004; 171:275-278.

16 Stein JP, Hertz J, Nichols PW: Urethral recurrence in a woman after continent orthotopic urinary diversion for bladder cancer. Urology 2008;71:755.e1-755.e2.

17 Jones J, Melchior SW, Gillitzer R, et al: Urethral recurrence of transitional cell carcinoma in a female patient after cystectomy and orthotopic ileal neobladder. J Urol 2000;164: 1646.
18 Gakis G, Efstathiou J, Lerner SP, et al: ICUDEAU International Consultation on Bladder Cancer 2012: radical cystectomy and bladder preservation for muscle-invasive urothelial carcinoma of the bladder. Eur Urol 2013;63:45-57.

19 Stein JP, Penson DF, Lee C, et al: Long-term oncological outcomes in women undergoing radical cystectomy and orthotopic diversion for bladder cancer. J Urol 2009;181:2052-2059.

20 Varol C, Thalmann GN, Burkhard FC, et al: Treatment of urethral recurrence following radical cystectomy and ileal bladder substitution. J Urol 2004;172:937-942.

21 Volkmer BG, Kuefer R, Bartsch GC, et al: Oncological follow up after radical cystectomy for bladder cancer - is there any benefit? J Urol 2009;181:1587-1593.

22 Giannarini G, Kessler TM, Thoeny HC, et al: Do patients benefit from routine follow-up to detect recurrences after radical cystectomy and ileal orthotopic bladder substitution? Eur Urol 2010;58:486-494.

23 Taylor JM, Spiess PE, Kassouf W, et al: Management of urethral recurrence after orthotopic urinary diversion. BJU Int 2010;106:56-61.

24 Yamashita S, Hoshi S, Ohyama C, et al: Urethral recurrence following neobladder in bladder cancer patients. Tohoku J Exp Med 2003;199:197-203.

-25 Yossepowitch O, Dalbagni G, Golijanin D, et al: Orthotopic urinary diversion after cystectomy for bladder cancer: implications for cancer control and patterns of disease recurrence. J Urol 2003;169:177-181.

26 Stenzl A, Jarolim L, Coloby P, et al: Urethrasparing cystectomy and orthotopic urinary diversion in women with malignant pelvic tumors. Cancer 2001;92:1864-1871. 